\title{
A study of three referral methods for wisdom patients
}

\author{
A controlled trial of three referral methods for patients with third molars. by R. D. Goodey, M. R. Brickley, C. M. Hill, \\ and J. P. Shepherd BrDent J 2000; 189:556-560
}

\section{Aim}

To evaluate the accuracy, sensitivity and specificity of three primary to secondary care referral strategies.

\section{Method \\ Thirty two primary care dental practitioners (GDPs) were randomly allocated one of three referral strategies: current practice (control strategy); a neural network embedded within a computer program and a paper-based clinical algorithm. One hundred and seven patients were assessed for lower third molar treatment: 47, 30 and 30 in each group, respectively. Clinical details were assessed by a panel of experts against a gold standard for third molar removal (the National Institutes of Health criteria). The accuracy, sensitivity, specificity, positive and negative predictive values were calculated for each strategy.}

\section{Results}

The referral decisions made by the GDPs in the control group displayed greater accuracy and sensitivity but poorer specificity $(0.83 ; 0.97 ; 0.22)$ compared with the neural network $(0.67 ; 0.56$; $0.79)$ and clinical algorithm $(0.73 ; 0.56 ; 0.93)$.

\section{Conclusions}

It was concluded that incorporation of the clinical algorithm into primary care was the most appropriate option. The computer neural network performed less well than either current practice or the clinical algorithm.

\section{Comment}

$\mathrm{T}$ his group have established a strong rep1 utation in the field of decision-making analysis and in particular the study of third molars.

This latest offering from the group looks at the referral of wisdom teeth to an oral surgery unit and asks whether the referral of appropriate cases can be improved upon. It takes as its gold-standard the National Institutes of Health criteria considered to be strong indications for third molar removal. The issue is that more informed referral should reduce unnecessary referrals, which would reduce pressure on clinics and avoid wasting the patients' time (allowing the hospital staff to concentrate on more worthy cases). The cost savings were estimated at $\mathfrak{1} 400 \mathrm{~K}$.

The purpose of this controlled trial was to evaluate the accuracy, sensitivity and specificity of three different referral strategies. Strategy 1 was their current practice, ie no change in what they considered suitable for referral. Strategy 2 involved the use of a neural network-based computer programme (clinical information is typed into the computer and the decision on whether to refer is then provided). Strategy 3 was a paper-based flow chart (clinical algorithm) which helped the dentist decide whether or not to refer the patient.

It is not clear over what time period the study took place, but 125 referral decisions from 20 dentists were made. Variation in referral decision was greatest within the control group, presumably because in the other two groups the novel aides to referral were more tightly defined. The two novel decision strategies made the same number of true positive and false negative decisions when compared with the NIH criteria. However, the clinical algorithm made more true negative referral decisions than the neural network. The control strategy, ie where it was left entirely up to the dentist whether to refer or not, was the most sensitive and accurate of the three decisionmaking strategies. However, they were also most likely to refer patients that did not require lower third molar surgery. In contrast the use of the novel-decision strategies, particularly the clinical algorithm, led to fewer unnecessary referrals for lower third molar surgery.

One particular problem was the apparent reluctance of the general dental practition- ers to participate (of 112 practitioners invited, only 32 were recruited). This may be a regional phenomenon as some studies have managed to recruit a great many practitioners, eg Tayside, ${ }^{1}$ whilst others have not, eg London region. ${ }^{2}$

Since the experience of those general dental practitioners who did recruit patients is known, it would have been interesting to see what influence this had on their referral decision within each strategy. The authors conclude that the clinical algorithm combined with existing practice is the best option in a general dental practice setting. Whether the refinement in correct referral pattern would lead to significant savings in dealing with inappropriate referrals is debatable. However, this pilot study serves as a useful reference point for further study.

\section{G. R. Ogden}

Professor of Oral \& Maxillofacial Surgery, University of Dundee Dental School

1 Ogden G R, Bissias E, Ruta D A. Quality of life following third molar removal: a patient versus professional perspective. Br Dent J 1998; 183: 407-410. 